\title{
Towards Ptychography with Structured Illumination, and a Derivative-Based Reconstruction Algorithm
}

W. Van den Broek, ${ }^{1 *}$ M. Schloz, ${ }^{1}$ T.C. Pekin, ${ }^{1}$ P.M. Pelz, ${ }^{2}$ P.-H. Lu, ${ }^{3}$ M. Kruth, ${ }^{3}$ V. Grillo, ${ }^{4}$ R.E. DuninBorkowski, ${ }^{3}$ R.J.D. Miller, ${ }^{2,5}$ C.T. Koch $^{1}$

1. Institut für Physik \& IRIS Adlershof, Humboldt-Universität zu Berlin, Berlin, Germany

2. Max Planck Institute for the Structure and Dynamics of Matter, Center for Free Electron Laser Science, Hamburg, Germany

3. Ernst Ruska-Centre (ER-C) for Microscopy and Spectroscopy with Electrons, Forschungszentrum Jülich, Jülich, Germany

4. CNR-Istituto Nanoscienze, Centro S3, Modena, Italy

5. Departments of Chemistry and Physics, University of Toronto, Toronto, Canada.

* Corresponding author: vandenbroek@physik.hu-berlin.de

The method inverse dynamical electron scattering (IDES) [1,2] offers a framework to retrieve the electrostatic potential of the object from arbitrary detection geometries in the transmission electron microscope (TEM). This has been illustrated on TEM simulations [1,2] and experimentally on an optical set-up [3]. Although ptychography was already included [2], now a specialized software has been developed: regularized optimization for ptychography (ROP). Contrary to the more conventional Gerchberg-Saxton formalism, ROP retrieves the potential, the electron probe and the probe positions through a derivative-based Polak-Ribiere conjugate gradients optimization, and is capable of regularizing the reconstruction. Furthermore, first results of the practical implementation of structured illumination are presented.

By approximating the scattering of the fast electrons in the solid with the multislice algorithm, multiple scattering in the forward direction is taken into account. Furthermore, an arbitrary error function can be defined, and the backpropagation algorithm, combined with the chain rule, enables efficient computation of the derivatives of said function with respect to the object potential, and arbitrary model parameters like amplitude, phase and position of the incoming beam; estimation of the latter parameters is especially important for structured illumination set-ups. The potential can be approximated through Dirac delta functions located at the atom positions and convoluted with a so-called generalized potential accounting for the average atom's width. Adding a regularization term (the sum of absolute values of the reconstructed potential) to the error function prevents overfitting and improves sparsity.

Structured illumination was obtained through a diffuser in the condenser plane of the microscope. Its phase modulation was realized through the mean inner potential of a thin film with spatial thickness variation. For production a 200-nm-thick silicon nitride (SiN) membrane over a 200- $\mu$ m-thick Si substrate was thinned using FEI Helios NanoLab 460F1 focused ion beam milling. A 150-nm-thick Au layer, acting as a diaphragm and reducing charging, was coated on the membrane surrounding the diffuser area. Analogous to [4], the diffuser was designed with a Gerchberg-Saxton algorithm iterating back and forth between real and reciprocal space; each time enforcing the respective constraints: in real space the intensity profile of the beam was set to a cosine of width $2 \mathrm{~nm}$ and in reciprocal space the intensity was set to 1 and 0 for angles below and above the Scherzer angle, respectively.

In Figure 1. the results from simulated diffraction patterns are shown. The sample is a [001] oriented Si- 
wedge, increasing in thickness from 0 to $15 \mathrm{~nm}$. CBED patterns were simulated with the multislice algorithm FDES [5], the acceleration voltage was $60 \mathrm{kV}$, the semi-convergence angle of the probe was $20 \mathrm{mrad}$, the probe was defocused by $3.7 \mathrm{~nm}$, the scan step was $0.066 \mathrm{~nm}$, and the slice thickness was $0.1 \mathrm{~nm}$. For the reconstruction the thickness was assumed known, the initial defocus was set to $7.7 \mathrm{~nm}$, the beam positions were given a random displacement away from the true values of $0.036 \mathrm{~nm}$ on average, and incorporation of the multislice formalism ensured a sufficiently accurate treatment of the multiple scattering with a slice thickness of $1 \mathrm{~nm}$ and all slices constraint to be equal. The object potential is reconstructed faithfully and the probe and probe positions are retrieved as well.

The diffuser was inserted in the probe-forming optics, specifically, the condenser 2 aperture plane, of the FEI Titan HOLO microscope at the Ernst Ruska-Centre (ER-C) in Jülich, Germany. As can be observed in Figure 2. at $1.9 \mathrm{~nm}$ the width of the experimental beam is reasonably close to the designed value of 2 $\mathrm{nm}$. Preliminary calculations attribute the more irregular contrast in the experimental probe to the presence of spherical aberration. [6]

\section{References:}

[1] W. Van den Broek and C.T. Koch, Phys. Rev. Lett. 109 (2012) p. 245502.

[2] W. Van den Broek and C.T. Koch, Phys. Rev. B 87 (2013) p. 184108.

[3] X. Jiang, W. Van den Broek and C.T. Koch, Optics Express 24 (2016) p. 7006.

[4] P.M. Pelz, et al., Scientific Reports 7 (2017) p. 9883.

[5] W. Van den Broek, X. Jiang and C.T. Koch, Ultramicroscopy 158 (2015) p. 89.

[6] Acknowledgments. WVDB: DFG project BR 5095/2-1. PHL and REDB: the German-Israeli Project cooperation grant from the German Research Foundation. VG: support by Q-SORT, funded by the

European Union's Horizon 2020 Research and Innovation Programme under grant agreement No. 766970 .
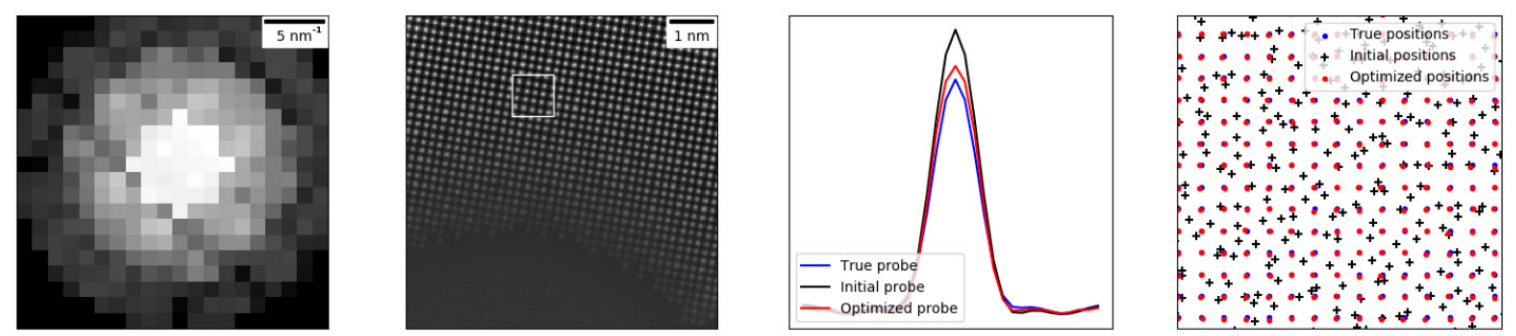

Figure 1. From left to right: a typical CBED pattern (logarithmic grayscale); reconstructed potential; profiles of initial, optimized and true probe; initial, optimized and true probe positions from the area in the second image, average deviation from true positions after optimization: $0.0036 \mathrm{~nm}$.
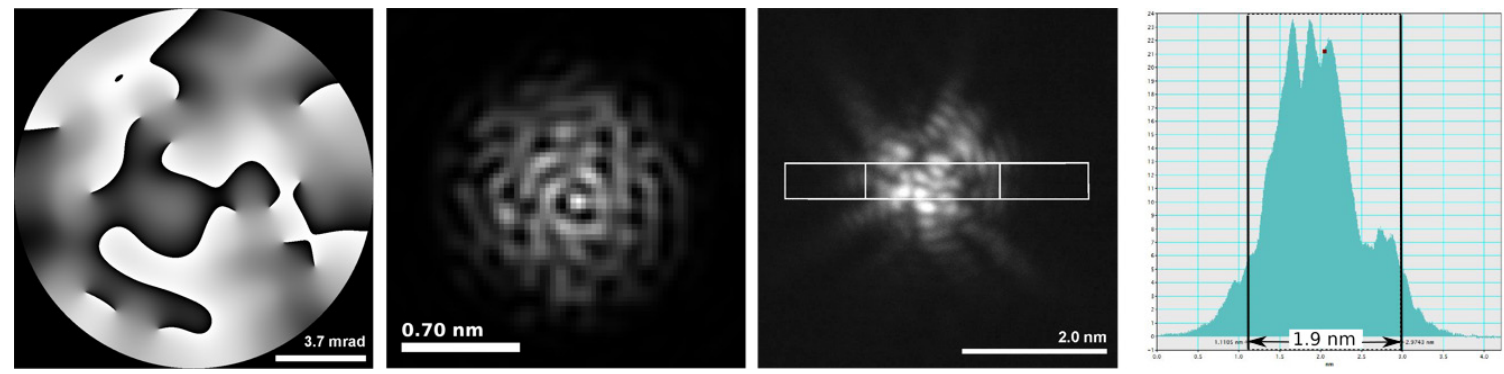

Figure 2. From left to right: design of the diffuser; nominal beam intensity computed from diffuser design; beam as experimentally realized; beam profile. 